\title{
Biliary Self-Expandable Metal Stent Could Be Recommended as a First Treatment Modality for Immediate Refractory Post-Endoscopic Retrograde Cholangiopancreatography Bleeding
}

\author{
Sun Young Moon ${ }^{1,2}$, Jun Heo ${ }^{1,2}$, Min Kyu Jung ${ }^{1,2}$, and Chang Min Cho $0^{1,2}$ \\ ${ }^{1}$ Department of Internal Medicine, Kyungpook National University Hospital, ${ }^{2}$ School of Medicine, Kyungpook National University, \\ Daegu, Korea
}

Background/Aims: Recent reports suggest that the biliary self-expandable metallic stent (SEMS) is highly effective for maintaining hemostasis when endoscopic hemostasis fails in endoscopic retrograde cholangiopancreatography (ERCP)-related bleeding. We compared whether temporary SEMS offers better efficacy than angioembolization for refractory immediate ERCP-related bleeding. Methods: Patients who underwent SEMS placement or underwent angioembolization for bleeding control in refractory immediate ERCP-related bleeding were included in the retrospective analysis. We evaluated the hemostasis success rate, severity of bleeding, change in hemoglobin levels, amount of transfusion, and delay to the start of hemostasis.

Results: A total of 27 patients with SEMS and 13 patients who underwent angioembolization were enrolled. More transfusions were needed in the angioembolization group ( $1.0 \pm 1.4$ units vs. $2.5 \pm 2.0$ units; $p=0.034)$. SEMS failure was successfully rescued by angioembolization. The partially covered SEMS ( $n=23,85.1 \%)$ was generally used, and the median stent-indwelling time was 4 days. The mean delay to the start of angioembolization was $95.2 \pm 142.9$ (range, 9-491) min.

Conclusions: Temporary SEMS had similar results to those of angioembolization ( $96.3 \%$ vs. $92.3 \%$; $p=0.588)$. Immediate SEMS insertion is considered a bridge treatment modality for immediate refractory ERCP-related bleeding. Angioembolization still has a role as rescue therapy when SEMS does not work effectively. Clin Endosc 2022;55:128-135

Key Words: Bleeding; Embolization; Endoscopic retrograde cholangiopancreatography; Self-expandable metallic stent

\section{INTRODUCTION}

Endoscopic retrograde cholangiopancreatography (ERCP) is a useful method for diagnosing and treating pancreatobiliary diseases. However, it may be associated with various complications. Bleeding is one of the most common adverse events associated with ERCP. The rate of post-ERCP bleeding is reportedly between $0.3 \%$ and $9.6 \%$ and depends on the

Received: January 28, 2021 Revised: March 23, 2021

Accepted: March 24, 2021

Correspondence: Jun Heo

Department of Internal Medicine, Kyungpook National University Hospital, 130 Dongdeok-ro, Jung-gu, Daegu 41944, Korea

Tel: +82-53-200-6504; Fax: +82-53-426-2046; E-mail: hero797@hanmail.net ORCID: https://orcid.org/0000-0003-3180-1655

(c) This is an Open Access article distributed under the terms of the Creative Commons Attribution Non-Commercial License (http://creativecommons.org/ licenses/by-nc/3.0) which permits unrestricted non-commercial use, distribution, and reproduction in any medium, provided the original work is properly cited. definition of bleeding. ${ }^{1,2}$ The severity of bleeding could be estimated by clinical significance: mild (no need for transfusion, hemoglobin drop of $<3 \mathrm{~g} / \mathrm{dL}$ ), moderate (transfusion of up to 4 units), and severe (transfusion of $>5$ units or need for intervention). ${ }^{3}$

When bleeding occurs during or immediately after ERCP, conventional endoscopic hemostasis, such as epinephrine injection, hemocoagulation, and balloon tamponade, is initially conducted. ${ }^{4-7}$ For cases that cannot be controlled by initial endoscopic hemostasis, angioembolization is conventionally used. ${ }^{8-10}$ However, there are some difficulties in angioembolization, in which time delay is inevitable and an experienced interventional radiologist is required. Recently, there have been a few case reports on the placement of endoscopic biliary self-expandable metallic stents (SEMS) to control refractory post-ERCP bleeding with excellent hemostasis outcomes with an almost $100 \%$ success rate., ${ }^{711,12}$ SEMS placement has a hemostatic effect by directly placing a mechanical tamponade 
against the hemorrhaging region. However, to date, no study has compared the efficacy of endoscopic biliary SEMS with angioembolization.

This study explores how SEMS may offer better efficacy than angioembolization when standard endoscopic management fails to achieve hemostasis in immediate ERCP-related bleeding.

\section{MATERIALS AND METHODS}

\section{Study design and patient selection}

\section{Patients}

We retrospectively reviewed the medical records of patients who had undergone ERCP or angioembolization at Kyungpook National University Hospital in Daegu, Republic of Korea, between January 2011 and May 2019. Data on patient characteristics, history, medications, procedure details, and clinical outcomes were reviewed. Patients with immediate ERCP-related bleeding that could not be controlled by initial endoscopic hemostasis were analyzed.

The patients were treated with either temporary SEMS placement or angioembolization. Immediate bleeding refers to bleeding that occurs during or immediately after the ERCP procedure. $^{13}$ This study was approved by the Institutional Review Board (approval number: KNUH 2020-03-005-003) and was conducted in accordance with the guidelines of the Declaration of Helsinki (1989 revision).

\section{Inclusion and Exclusion criteria}

The inclusion criteria were as follows: (1) patients who underwent ERCP and underwent SEMS insertion for bleeding control (SEMS group) and (2) patients who underwent embolization of abdominal vessels for hemostasis of post-ERCP bleeding (angioembolization group). If SEMS failed to control post-ERCP bleeding control and angioembolization was performed immediately afterward, the case was included in the SEMS group and classified as SEMS failure.

The exclusion criteria were as follows: (1) patients who underwent SEMS and angioembolization for other purposes and (2) cases of delayed ERCP-related bleeding. Delayed bleeding was defined as bleeding occurring more than $6 \mathrm{~h}$ after ERCP.

A total of 5,717 patients underwent ERCP during the study period; 426 underwent endoscopic biliary SEMS, of whom 28 had biliary metallic stent insertion for bleeding control. After excluding one patient with delayed bleeding, 27 patients were included in the SEMS group. A total of 1,092 patients received embolization for abdominal vessels, 18 of whom also received ERCP during the same hospital stay. Except for four cases of delayed bleeding, one case of no bleeding focus on angiography, and one case of rescue for SEMS failure, all patients were enrolled in the angioembolization group. In addition, one patient who had undergone initial ERCP at an outside hospital who was transferred to our hospital for angioembolization to treat refractory post-ERCP bleeding was also included. Finally, 13 patients were treated with angioembolization (angioembolization group) (Fig. 1).

\section{ERCP procedure and in-hospital course}

ERCP was performed during hospitalization with close monitoring and follow-up. All ERCP procedures were performed by two experienced pancreatobiliary specialists. A nurse-administered triple combination (midazolam, propofol, and pethidine) was used for sedation, and a TJF-260V (Olympus, Tokyo, Japan) endoscope was used for examination.

The vital signs of all patients were checked, and all patients were subjected to laboratory and imaging tests. Laboratory tests, including complete blood count, liver function test, amylase, lipase, and abdominal plain imaging, were performed before and after ERCP. Post-ERCP laboratory tests and abdominal plain imaging were performed twice, 4-6 hours after ERCP and on the morning of the next day. Vital signs and signs of gastrointestinal bleeding, such as melena, hematochezia, and hematemesis, were closely monitored during hospitalization.

\section{Conventional endoscopic control of bleeding}

Initial conventional endoscopic hemostasis included epinephrine injection, balloon tamponade, hemocoagulation, and hemoclipping. ${ }^{4-7}$ We usually used $0.5-4 \mathrm{~mL}$ diluted epinephrine (1:10,000) administered using a 23-gauge endoscopic sclerotherapy needle (TeleMed system, Hudson, MA, USA) or a retrieval balloon catheter into and around the sphincterotomy site. Balloon tamponade with a 15-mm-diameter balloon catheter (Escort II; Cook Medical, Bloomington, IN, USA) was used at the sphincterotomy site, and endoclips were sometimes used at the bleeding site. When the bleeding focus was identified, hemocoagulation was performed using an electrocoagulation probe (MTW, Wesel, Germany). These therapies can be used as monotherapy or in combination with other hemostatic methods. The selection of the initial conventional hemostatic methods was decided by the endoscopists.

\section{SEMS insertion and removal}

For SEMS insertion, after guidewire insertion, the SEMS was placed across the ampullary orifice and distal common bile duct (CBD), which could directly tamponade the bleeding site. After hemostasis was achieved with an endoscopic biliary SEMS, a second-look ERCP was scheduled within 1 week 

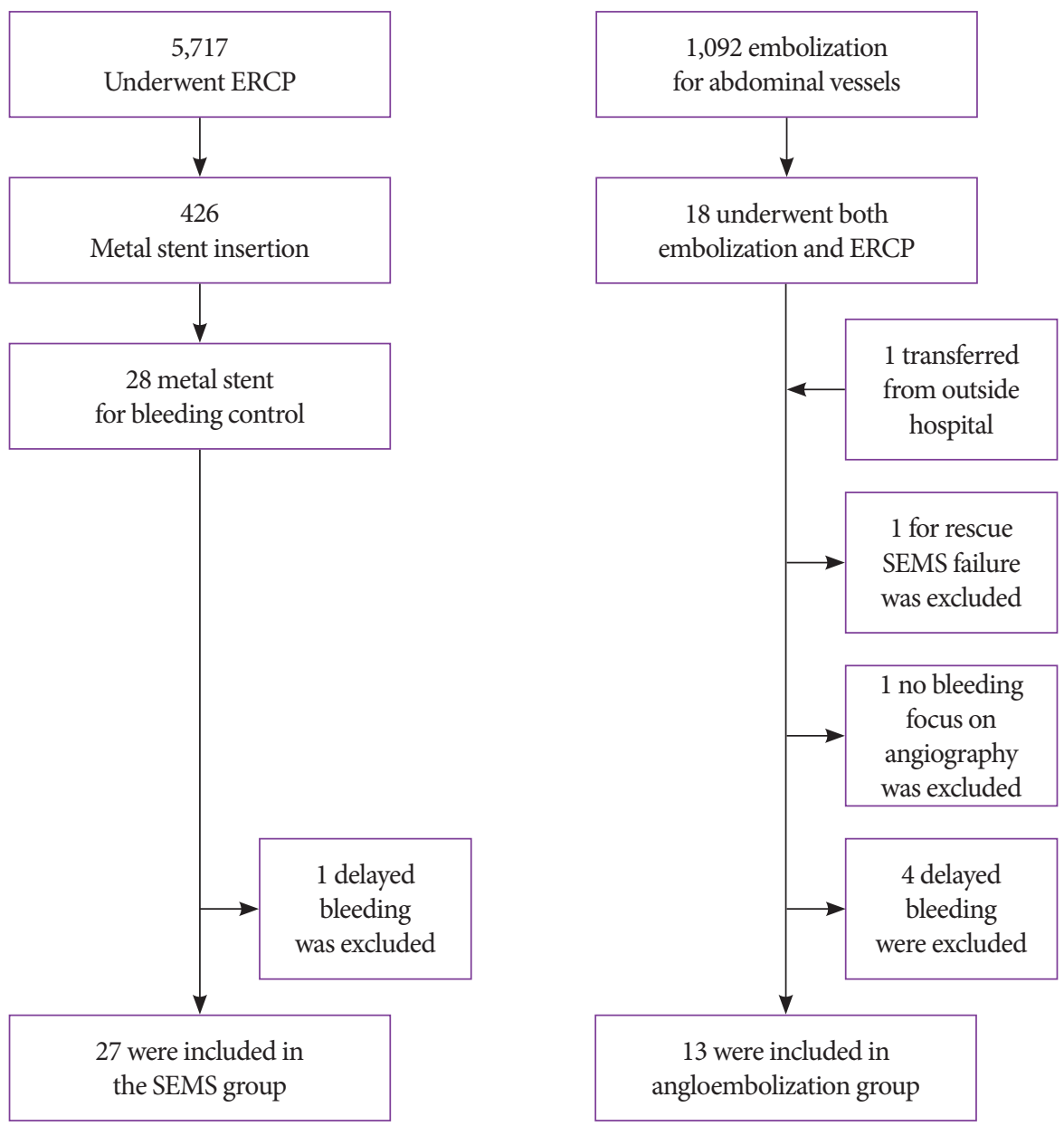

Fig. 1. Participant flow diagram. ERCP, endoscopic retrograde cholangiopancreatography; SEMS, Self-expandable metal stent.

after placement. If definite hemostasis was confirmed during the second-look ERCP, the previous stent was gently removed using rat-toothed forceps. After biliary stent removal, the presence of further complications was checked using cholangiogram and duodenoscopy.

\section{Angioembolization}

Angioembolization was used as a rescue therapy when massive bleeding obscured the endoscopic field during ERCP or when SEMS placement for tamponade failed to achieve hemostasis. Angioembolization was performed by two experienced interventional radiologists. Initially, angiographic procedures were performed using standard percutaneous transfemoral catheterization. After gaining ultrasound-guided access through the right common femoral artery to the celiac artery and superior mesenteric artery, angiography using a 5-Fr diagnostic catheter was performed to evaluate the vascular anatomy and identify the bleeding site. If a bleeding sign was noted, selective angiography was performed using a microcatheter. Superselective catheterization is preferred to prevent organ ischemia. Gelatin sponge, microcoil, glue, and lipiodol mixture were used as embolic materials. Completion angiography was performed to confirm the absence of further bleeding. The vital signs of all patients were checked, and they underwent laboratory tests, including complete blood count, liver function test, amylase, and lipase after the procedure. Post-ERCP laboratory tests and abdominal plain imaging were performed twice, 4-6 hours after ERCP and on the morning of the next day. Vital signs and signs of gastrointestinal bleeding, such as melena, hematochezia, and hematemesis, were closely moni- 
tored during hospitalization.

\section{Study outcome}

We evaluated the ERCP procedure time, ERCP-related pancreatitis, other ERCP-related complications, severity of bleeding, causes of immediate post-ERCP bleeding, hemostasis success rate, change in hemoglobin level, blood transfusion amount, length of hospital stay, bleeding-related mortality, and time to the start of hemostasis. The primary outcome was hemostasis success rate, and secondary outcomes were ERCP-related complications, severity of bleeding, cause of bleeding, change in hemoglobin level, blood transfusion amount, length of hospital stay, bleeding-related mortality, and time to the start of hemostasis.

\section{Statistical analysis}

SPSS (version 23 for Windows; IBM Co., Armonk, NY,
USA) was used for all statistical analyses. Statistical analysis of the results was performed using the chi-square test, Student's $t$-test, and Fisher's exact test. Statistical significance was set at $p<0.05$.

\section{RESULTS}

\section{Characteristics of patients}

In terms of baseline characteristics, the patients in the SEMS and angioembolization groups showed no significant difference in age $(72.5 \pm 13.7,72.3 \pm 11.6$, respectively; $p=0.962)$ or sex ratio (male, 15 [55.6\%] vs. 7 [53.8\%]; $p=0.919$ ). The SEMS group had more comorbidities, but the difference was not statistically significant (Charlson comorbidity index $4.2 \pm 1.8$, $3.2 \pm 1.2 ; p=0.070)$. The reason for ERCP was not statistically

Table 1. Baseline Characteristics of Patients

\begin{tabular}{|c|c|c|c|}
\hline & SEMS $(n=27)$ & Angioembolization $(n=13)$ & $p$-value \\
\hline Age at ERCP (years) & $72.5 \pm 13.7$ & $72.3 \pm 11.6$ & 0.962 \\
\hline Sex & & & 0.919 \\
\hline - Male & $15(55.6)$ & $7(53.8)$ & \\
\hline - Female & $12(44.4)$ & $6(46.2)$ & \\
\hline CCI & $4.2 \pm 1.8$ & $3.2 \pm 1.2$ & 0.070 \\
\hline Reason of ERCP & & & 0.176 \\
\hline - Stone & $21(77.8)$ & $10(76.9)$ & \\
\hline - PB malignancy & $2(7.4)$ & $3(23.1)$ & \\
\hline - Benign stricture & $4(14.8)$ & $0(0.0)$ & \\
\hline Bleeding risk factors & & & 1.000 \\
\hline - Liver cirrhosis & $3(11.1)$ & $1(7.7)$ & \\
\hline - Antiplatelet, antithrombotics & $7(25.9)$ & $3(23.1)$ & \\
\hline - none & $17(63.0)$ & $9(69.2)$ & \\
\hline PT (sec) & $11.8 \pm 1.6$ & $13.4 \pm 3.0$ & 0.039 \\
\hline aPTT (sec) & $26.9 \pm 5.5$ & $30.4 \pm 5.2$ & 0.065 \\
\hline $\mathrm{WBC}\left(10^{3} / \mu \mathrm{L}\right)$ & $9280.7 \pm 5730.6$ & $9752.3 \pm 4216.6$ & 0.794 \\
\hline $\mathrm{Hb}(\mathrm{g} / \mathrm{dL})$ & $12.0 \pm 1.9$ & $11.2 \pm 2.7$ & 0.280 \\
\hline Platelet $\left(10^{3} / \mu \mathrm{L}\right)$ & $212.3 \pm 114.6$ & $236.1 \pm 232.8$ & 0.665 \\
\hline Total bilirubin (mg/dL) & $3.6 \pm 4.9$ & $7.0 \pm 11.3$ & 0.195 \\
\hline $\operatorname{AST}(\mathrm{U} / \mathrm{L})$ & $173.3 \pm 128.3$ & $188.0 \pm 125.6$ & 0.440 \\
\hline $\operatorname{ALT}(\mathrm{U} / \mathrm{L})$ & $124.5 \pm 127.5$ & $127.9 \pm 154.6$ & 0.942 \\
\hline
\end{tabular}

Data are presented as the number (\%) or mean \pm standard deviation.

ALT, alanine transaminase; aPTT, activated partial thromboplastin time; AST, aspartate aminotransferase; CCI, Charlson comorbidity index; ERCP, endoscopic retrograde cholangiopancreatography; Hb, hemoglobin; PB, pancreatobiliary; PT, prothrombin time; SEMS, self-expandable metal stent; WBC, white blood cell. 
significantly different between the two groups (choledocholithiasis, $n=21$ [77.8\%] vs. 10 [76.9\%]; pancreatobiliary malignancy, $n=2$ [7.4\%] vs. 3 [23.1\%]; and benign stricture, $n=4[14.8 \%]$ vs. $0[0.0 \%] ; p=0.176)$. No significant difference was observed in the medication of antithrombotic agents, liver cirrhosis, thrombocytopenia, or initial hemoglobin level before the ERCP procedure in the SEMS and angioembolization groups ( $12.0 \pm 1.9$ vs. $11.2 \pm 2.7 ; p=0.280$ ). However, prothrombin time was higher in the angioembolization group $(11.8 \pm 1.6$ vs. $13.4 \pm 3.0 ; p=0.039)$ (Table 1$)$.

\section{ERCP-related results}

ERCP procedure time $(23.8 \pm 7.4 \mathrm{~min}$ vs. $21.4 \pm 7.3 \mathrm{~min}$; $p=0.336)$ and the rate of post-ERCP pancreatitis (0 [0\%] cases vs. $1[7.7 \%]$ case; $p=0.144)$ did not differ between the two groups. Regarding the cause of post-ERCP bleeding, bleeding related to endoscopic sphincterotomy occurred in 21 cases; 15/21 (72.4\%) were moderate-sized incisions and 6/21 (27.6\%) were full-sized incisions. In patients with malignancy or strictures, 2/21 (9.95\%) cases had hemostasis performed simultaneously by using SEMS, and 3/21 (14.2\%) cases required immediate angioembolization to control bleeding and maintain patency following massive hemorrhage that precluded us from localizing the bleeding focus or that resulted in unstable vital signs. With the exception of such circumstances, we at- tempted two or more methods of endoscopic hemostasis. In cases of bleeding related to endoscopic papillary large balloon dilation (EPLBD), we performed SEMS or angioembolization in 4/15 (26.78\%) cases in which massive bleeding occluded the visualization and localization of the bleeding focus. Balloon tamponade was attempted in 10/15 (66.7\%) cases, and a combination of epinephrine spray, balloon tamponade, and hemocoagulation, followed by SEMS or angioembolization due to failure of hemostasis, was performed in 1/15 (6.7\%) cases. Shock before the ERCP procedure (3 [11.1\%] vs. 2 [15.4\%]; $p=0.702)$ and use of inotropics for shock $(3[11.1 \%]$ vs. 3 [23.17\%]; $p=0.321$ ) were slightly, but not significantly, more common in the angioembolization group (Table 2).

\section{Type and duration of stent}

In the SEMS group, 23 cases (85.1\%) involved a partially covered stent (Niti-S ComVi, Taewoong Medical, Gimpo, Korea), one involved a fully covered stent (Niti-S, pyloric; Taewoong Medical, Gimpo, Korea), one involved a fully covered type (Niti-S, Bumpy type; Taewoong Medical, Gimpo, Korea), and two cases involved a fully covered stent (WallFlex; Boston Scientific Corp., Marlborough, MA, USA). The mean diameter of the $\mathrm{CBD}$ was $13.3 \pm 3.4 \mathrm{~mm}$. Stent diameter and length were selected by the clinician who performed the procedure based on the CBD diameter and the presence of other condi-

Table 2. Endoscopic Retrograde Cholangiopancreatography-Related Clinical Outcomes

\begin{tabular}{lccc}
\hline & SEMS $(\boldsymbol{n = 2 7})$ & Angioembolization $(\boldsymbol{n}=\mathbf{1 3})$ & $\boldsymbol{p}$-value \\
\hline ERCP procedure time (min) & $23.8 \pm 7.4$ & $21.4 \pm 7.3$ & 0.336 \\
Post-ERCP pancreatitis cases & $0(0)$ & $1(7.7)$ & 0.144 \\
Bleeding severity & & $3(23.1)$ & 0.006 \\
- Mild (no transfusion) & $15(55.6)$ & $6(46.2)$ & $4(30.8)$ \\
- Moderate (upto 4 units) & $12(44.4)$ & $2(15.4)$ & 0.702 \\
- Severe (>5 units) & $0(0.0)$ & $3(23.1)$ & 0.321 \\
Shock, before ERCP & $3(11.1)$ & $1(7.7)$ & 0.974 \\
Using inotropics & $3(11.1)$ & & 0.792 \\
Other ERCP-related complications & $2(7.4)$ & $6(46.2)$ & $6(46.2)$ \\
Bleeding during ERCP & & $1^{\text {b) }}(7.7)$ & \\
- Post-ES bleeding & $15(55.6)$ & $9(33.3)$ & \\
- Post-EPLBD bleeding & $3^{\text {a) }}(11.1)$ & & \\
- Other & &
\end{tabular}

Data are presented as the number (\%) or mean \pm standard deviation.

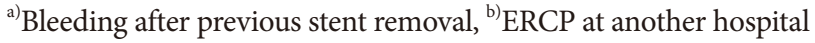

EPLBD, endoscopic papillary large balloon dilation; ERCP, endoscopic retrograde cholangiopancreatography; ES, endoscopic sphincterotomy; SEMS, self-expandable metallic stents. 
Table 3. Clinical Outcomes in the Two Groups

\begin{tabular}{lccc}
\hline & SEMS $(\boldsymbol{n = 2 7})$ & Angioembolization $(\boldsymbol{n}=\mathbf{1 3})$ & $\boldsymbol{p}$-value \\
\hline Hemostasis success rate (\%) & 96.3 & 92.3 & 0.588 \\
Changes in Hb level (g/dL) & $1.7 \pm 1.2$ & $1.9 \pm 1.7$ & 0.789 \\
Pack-RBC transfusion (units) & $1.0 \pm 1.4$ & $2.5 \pm 2.0$ & 0.034 \\
In-hospital stay (days) & $28.2 \pm 88.6$ & $14.9 \pm 7.3$ & 0.593 \\
Bleeding-related mortality & $0(0)$ & $1(7.7)$ & 0.144 \\
Migration & $1(3.7)$ & $0(0)$ & 0.482 \\
Cholecystitis & $0(0)$ & $0(0)$ & 0 \\
Pancreatitis & $0(0)$ & $1(7.7)$ & 0.144 \\
\hline
\end{tabular}

Data are presented as the number (\%) or mean \pm standard deviation.

ERCP, endoscopic retrograde cholangiopancreatography; Hb, hemoglobin; RBC, red blood cell; SEMS, self-expandable metallic stents.

tions, such as stricture or malignancy. We controlled bleeding by using a self-expandable stent with a $20-\mathrm{mm}$ diameter (pyloric/duodenal stent) to achieve effective tamponade in cases of bleeding after EPLBD or biliary duct dilatation. The median diameter of the SEMSs was $20 \mathrm{~mm}$ (range, 8-20 mm), and the median length was $80 \mathrm{~mm}$ (range, $50-100 \mathrm{~mm}$ ). No complications, such as cholecystitis or perforation, were observed. Only one spontaneous migration with successful hemostasis was reported 5 days after stent placement (partially covered; stent diameter, $10 \mathrm{~mm}$; length, $50 \mathrm{~mm}$ ).

After hemostasis of post-ERCP bleeding, SEMSs were usually removed within 3-4 days after the procedure. In this study, the median stent-indwelling time was 4 days (range, 1-122 days). There were four cases in which the metallic stent was retained for the treatment of biliary stricture after bleeding control, with two cases of benign biliary stricture and two cases of CBD cancer with obstruction. ${ }^{14}$ Excluding these cases, the median stent-indwelling time was 3 (range, 1-7) days.

\section{Clinical outcomes}

The hemostasis success rates were similarly high in both groups (26 [96.3\%] vs. 12 [92.3\%]; $p=0.588$ ). The duration of in-hospital stays was slightly longer for the SEMS group but was not statistically significant $(28.2 \pm 88.6$ vs. $14.9 \pm 7.3$ days; $p=0.593)$. In the SEMS group, one patient showed 471 days of in-hospital stay due to retroperitoneal perforation, operation-related infection, and deconditioning, which prolonged the length of hospital stay (Table 3). Excluding this case, the duration of in-hospital stays was longer for the angioembolization group with a statistical significance $(11.2 \pm 5.2$ days vs. $14.9 \pm 7.3$ days; $p=0.079$ ).

SEMS deployment was performed directly immediately after awareness of refractory ERCP-related bleeding; however, the mean time interval between ERCP and angioembolization was $95.2 \pm 142.9 \mathrm{~min}(p=0.022)$. The change in mean hemoglobin level, which means the difference between the initial hemoglobin level and lowest hemoglobin level after the ERCP procedure, were not different between the SEMS and angioembolization groups $(1.7 \pm 1.2 \mathrm{~g} / \mathrm{dL}$ vs. $1.9 \pm 1.7 \mathrm{~g} / \mathrm{dL}$; $p=0.789)$. However, more transfusions were needed in the angioembolization group (1.0 \pm 1.4 units vs. $2.5 \pm 2.0$ units; $p=0.034$ ), and ERCP-related bleeding was more serious in the angioembolization group (mild, $55.6 \%$ vs. $23.1 \%$; moderate, $44.4 \%$ vs. $46.2 \%$; severe, $0 \%$ vs. $30.8 \%$; $p=0.006$ ).

There was one case of SEMS failure, and angioembolization was performed for rescue therapy after SEMS deployment. An 89-year-old man with a Charlson comorbidity index of 4 had no risk factors for bleeding tendency underwent ERCP for a $\mathrm{CBD}$ stone. Active oozing persisted after SEMS placement, angioembolization was successfully performed to achieve hemostasis.

\section{DISCUSSION}

When conventional endoscopic hemostasis fails in immediate ERCP-related bleeding, persistent bleeding may result in a large amount of blood loss, culminating in a life-threatening situation. Traditionally, angioembolization is considered an alternative therapy in these cases. ${ }^{8,10}$ In our study, angioembolization was chosen in patients with massive hemorrhage, which precluded us from localizing the bleeding focus in those with bleeding noted $6 \mathrm{~h}$ after ERCP or in those who were unable to undergo ERCP due to hypoxia. However, the relatively 
high rebleeding rate due to the rich vascular network of the duodenum and the possibility of ischemic mucosal injury of the duodenum are major complications of angioembolization. ${ }^{8}$ Therefore, complete embolization could not be performed in cases lacking or with minimal extravasation. Moreover, the lack of expert interventional radiologists or angiographic units leaves patients with no choice but to be transferred to another hospital while they have persistent active bleeding. This overdue angioembolization may reduce the chances of survival. A 74-year-old male patient with a Charlson comorbidity index of 5 underwent initial ERCP because of suspected hilar cholangiocarcinoma at an outside hospital and was transferred to our hospital for angioembolization. The time between the initial ERCP and angioembolization was $6 \mathrm{~h}$ and $18 \mathrm{~min}$. This case was the only mortality in our study, despite performing angioembolization.

Biliary stents have a specific advantage in that they can be placed immediately after the failure of conventional endoscopic hemostasis. ${ }^{11,12}$ If conventional endoscopic hemostasis is continued for refractory ERCP bleeding, the total ERCP procedural time can be prolonged. In addition, immediate placement of the SEMS could save time by avoiding further conventional hemostasis. The change in hemoglobin levels was similar to the significant lower transfusion volume after ERCP in the SEMS group $(1.7 \pm 1.2 \mathrm{~g} / \mathrm{dL}$ vs. $1.9 \pm 1.7 \mathrm{~g} / \mathrm{dL}$; $p=0.789 ; 1.0 \pm 1.4$ units vs. $2.5 \pm 2.0$ units; $p=0.034$ ). The use of inotropics was slightly common in the angioembolization group, but the difference was not statistically significant. In summary, SEMS placement has the advantage of prompt treatment for refractory post-ERCP bleeding. These time savings would decrease the amount of bleeding and the amount of transfusion. Thus, mild bleeding was more dominant in the SEMS group ( $p=0.006$ ). Since January 2020, the Korea National Health Insurance Service has allowed coverage of metal stent usage in cases of biliary stricture bleeding that is resistant to conventional methods of hemostasis or difficult to stop for technical reasons, giving the modality a selective advantage in terms of cost.

Although SEMS placement is appropriate for transampullary and distal CBD, post-ERCP bleeding might not be controlled in some cases. ${ }^{7}$ There was one case of treatment failure using SEMS for hemostasis in our study. We believe that the tamponade pressure of the stent was not sufficient to stop bleeding in the periampullary branch of the duodenal artery. This patient was successfully treated with angioembolization. Therefore, angioembolization still plays a role in rescue therapy in cases of SEMS failure. In addition, in cases of refractory ERCP-related bleeding from far above the ampulla or distal $\mathrm{CBD}$, such as at the hilar level, angioembolization is consid- ered the first option for hemostasis.

There is no consensus on what kind of stent should be used or when it should be removed. Small-diameter SEMSs may not only migrate or dislocate, but may also provide insufficient tamponade to achieve hemostasis. ${ }^{12}$ In our study, we used a partially covered metallic stent $(n=23,85.1 \%)$. Owing to the advantage of partially covered metallic stents, we only had one case of migration in 23 partially covered SEMSs. The diameter of the migrated stent was $10 \mathrm{~mm}$, and it was deployed in a moderately dilated biliary duct, which was estimated to be approximately $10 \mathrm{~mm}$ in diameter. Stent migration might have occurred because the diameter of the stent was small relative to larger CBD diameter. The median diameter of the SEMSs was $20 \mathrm{~mm}$ (range, $8-20 \mathrm{~mm}$ ), and the median length was 80 $\mathrm{mm}$ (range, $50-100 \mathrm{~mm}$ ). The hemostatic effect is immediate, and the stent works through a tamponade effect; stents can be safely removed less than a week after deployment. ${ }^{12}$ In our study, the median indwelling time of all stents was 4 (range, 1-122) days. If we excluded the four cases of prolonged placement due to benign or malignant CBD stricture, the median indwelling time was 3 (range, 1-7) days. ${ }^{14,15}$ In summary, partially covered SEMS with a diameter larger than the CBD diameter could be recommended for refractory post-ERCP bleeding. In addition, removing the stent within 3-4 days after stent insertion for hemostasis is reasonable.

The relatively small study cohort and retrospective study design are the main limitations of our study. However, our results are noteworthy as they are the first to compare biliary SEMS and angioembolization for hemostasis after ERCP. In addition, in a life-threatening bleeding situation, a prospective study design is not possible because of ethical issues. Based on our results, a future prospective study design, which includes initial biliary stent placement and rescue angioembolization, could be evaluated as a standard management method for post-ERCP refractory bleeding control.

In conclusion, temporary deployment of biliary SEMS results in successful and durable hemostasis comparable to the results achieved with angioembolization. Our results indicate that temporary placement of SEMS could be a bridge treatment modality for immediate ERCP-related bleeding. In addition, traditional angioembolization still plays a role as a rescue therapy if SEMS hemostasis fails.

Conflicts of interest

The authors have no potential conflicts of interest.

Funding

None. 


\author{
Author Contributions \\ Conceptualization: Jun Heo, Minkyu Jung, Chang Min Cho \\ Data curation: Sun Young Moon \\ Formal analysis: SYM \\ Investigation: SYM, JH, MKJ, CMC \\ Methodology: SYM, JH, MKJ, CMC \\ Project administration: $\mathrm{JH}$ \\ Resources: JH, MKJ, CMC \\ Supervision: JH, MKJ \\ Validation: JH, MKJ, CMC \\ Visualization: SYM \\ Writing-original draft: SYM \\ Writing-review\&editing: SYM, JH, MKJ, CMC
}

\section{ORCID}

Sun Young Moon

Jun Heo

Min Kyu Jung

Chang Min Cho

\begin{abstract}
https://orcid.org/0000-0001-6610-5129 https://orcid.org/0000-0003-3180-1655 https://orcid.org/0000-0001-8749-408X https://orcid.org/0000-0002-9903-1282
\end{abstract}

\section{REFERENCES}

1. Dumonceau JM, Kapral C, Aabakken L, et al. ERCP-related adverse events: European Society of Gastrointestinal Endoscopy (ESGE) Guideline. Endoscopy 2020;52:127-149.

2. Cotton PB, Eisen GM, Aabakken L, et al. A lexicon for endoscopic adverse events: report of an ASGE workshop. Gastrointest Endosc 2010;71:446-454.

3. Cotton PB, Lehman G, Vennes J, et al. Endoscopic sphincterotomy complications and their management: an attempt at consensus. Gastrointest Endosc 1991;37:383-393.

4. Ferreira LE, Baron TH. Post-sphincterotomy bleeding: who, what, when, and how. Am J Gastroenterol 2007;102:2850-2858.
5. Wilcox CM, Canakis J, Mönkemüller KE, Bondora AW, Geels W. Patterns of bleeding after endoscopic sphincterotomy, the subsequent risk of bleeding, and the role of epinephrine injection. Am J Gastroenterol 2004;99:244-248.

6. Rustagi T, Jamidar PA. Endoscopic retrograde cholangiopancreatography-related adverse events: general overview. Gastrointest Endosc Clin N Am 2015;25:97-106.

7. Canena J, Liberato M, Horta D, Romão C, Coutinho A. Short-term stenting using fully covered self-expandable metal stents for treatment of refractory biliary leaks, postsphincterotomy bleeding, and perforations. Surg Endosc 2013;27:313-324.

8. So YH, Choi YH, Chung JW, Jae HJ, Song SY, Park JH. Selective embolization for post-endoscopic sphincterotomy bleeding: technical aspects and clinical efficacy. Korean J Radiol 2012;13:73-81.

9. Dunne R, McCarthy E, Joyce E, et al. Post-endoscopic biliary sphincterotomy bleeding: an interventional radiology approach. Acta Radiol 2013;54:1159-1164.

10. Maleux G, Bielen J, Laenen A, et al. Embolization of post-biliary sphincterotomy bleeding refractory to medical and endoscopic therapy: technical results, clinical efficacy and predictors of outcome. Eur Radiol 2014;24:2779-2786

11. Shah JN, Marson F, Binmoeller KF. Temporary self-expandable metal stent placement for treatment of post-sphincterotomy bleeding. Gastrointest Endosc 2010;72:1274-1278.

12. Itoi T, Yasuda I, Doi S, Mukai T, Kurihara T, Sofuni A. Endoscopic hemostasis using covered metallic stent placement for uncontrolled post-endoscopic sphincterotomy bleeding. Endoscopy 2011;43:369-372.

13. Chandrasekhara V, Khashab MA, Muthusamy VR, et al. Adverse events associated with ERCP. Gastrointest Endosc 2017;85:32-47.

14. Behm B, Brock A, Clarke BW, et al. Partially covered self-expandable metallic stents for benign biliary strictures due to chronic pancreatitis. Endoscopy 2009;41:547-551.

15. Schmidt A, Pickartz T, Lerch MM, et al. Effective treatment of benign biliary strictures with a removable, fully covered, self-expandable metal stent: a prospective, multicenter European study. United European Gastroenterol J 2017;5:398-407. 